\title{
Review
}

\section{Potential benefits and hazards of physical activity and exercise on the gastrointestinal tract}

\begin{abstract}
Summary
This review describes the current state of knowledge on the hazards of exercise and the potential benefits of physical activity on the gastrointestinal tract. In particular, acute strenuous exercise may provoke gastrointestinal symptoms such as heartburn or diarrhoea. A substantial part $(20-50 \%)$ of endurance athletes are hampered by these symptoms which may deter them from participation in training and competitive events. Nevertheless, these acute symptoms are transient and do not hamper the athlete's health in the long term. The only exception is repeated gastrointestinal bleeding during training and competition, which in the long term may occasionally lead to iron deficiency and anaemia. In contrast, repetitive exercise periods at a relatively low intensity may have protective effects on the gastrointestinal tract. There is strong evidence that physical activity reduces the risk of colon cancer by up to $50 \%$. Less convincing evidence exists for cholelithiasis and constipation. Physical activity may reduce the risk of diverticulosis, gastrointestinal haemorrhage, and inflammatory bowel disease although this cannot be substantiated firmly. Up to now, underlying mechanisms are poorly understood although decreased gastrointestinal blood flow, neuroimmuno-endocrine alterations, increased gastrointestinal motility, and mechanical bouncing during exercise are postulated. Future research on exercise associated digestive processes should give more insight into the relationship between physical activity and the function of the gastrointestinal tract.
\end{abstract}

\section{Introduction}

The impact of exercise and physical activity on the gastrointestinal tract is an area of emerging interest. During the past two decades research was mainly directed towards the hazards of strenuous exercise, especially gastrointestinal symptoms. ${ }^{1-3}$ In recent years, however, interest has also focussed on the potential benefits of physical activity on the gastrointestinal tract. Several studies indicate an inverse relationship between physical activity and risk of gastrointestinal related diseases such as colon cancer, ${ }^{4-6}$ diverticular disease, ${ }^{7}$ cholelithiasis, ${ }^{89}$ or constipation. ${ }^{10}$ While the prevalence of these diseases is relatively high and increases with age, participation in physical activity is relatively low and decreases with age.

This review summarises the current state of knowledge on the hazards of exercise and the potential benefits of physical activity. "Exercise" is considered as voluntary activation of skeletal muscle leading to short term effects (for minutes or hours) while "physical activity" is considered as repetitive exercise periods leading to long term effects (for days, weeks, months, or years). We focus on the role of physical activity in the prevention of several diseases of the gastrointestinal tract and the postulated mechanisms by which physical activity influences the gastrointestinal tract. These mechanisms will be discussed more extensively at the end of this review. As potential hazards of exercise have been the subject of several reviews (for example, see Moses, ${ }^{11}$ Peters et al, ${ }^{12}$ and Brouns and Beck$\mathrm{ers}^{13}$ ), they will be discussed only briefly.
The role of physical activity in the treatment of gastrointestinal diseases will not be discussed, as the literature on this topic is scarce. Nevertheless, in patients with gastrointestinal diseases physical activity may inhibit muscle loss, and improve appetite, functional capacity, and general well being by positive mood changes ${ }^{5}$.

\section{Hazards}

Gastrointestinal symptoms such as nausea, heartburn, diarrhoea, and gastrointestinal bleeding are common during exercise, especially during vigorous sports such as long distance running and triathlons. ${ }^{213-16}$ In general, these symptoms are transient and can be considered protective for critical organ damage: its progressive nature causes the athlete to reduce exercise intensity or duration. Sometimes the symptoms may be so serious that they can limit exercise performance severely ${ }^{1216}$ and even participation in physical activity. ${ }^{12}$

Incidence rates during prolonged exercise vary mostly from $20 \%$ to $50 \%$, depending on factors such as mode, duration, and intensity of exercise, type of symptom, age, training status, sex, dietary intake, occurrence of gastrointestinal symptoms at rest, and method of investigation. ${ }^{12} 1315$ In particular, exercise intensity seems to be an important factor provoking gastrointestinal symptoms. ${ }^{121316}$ The mechanisms by which exercise causes gastrointestinal symptoms are not well known. Decreased gastrointestinal blood flow, increased gastrointestinal motility, increased mechanical bouncing, and altered neuroendocrine modulation are postulated. ${ }^{11-13}$ All of these mechanisms are associated with exercise intensity. ${ }^{13}{ }^{17-20}$

While most gastrointestinal symptoms do not hamper the athlete's health, gastrointestinal bleeding may be a serious problem. Most often the type of bleeding is occult and transient, although anecdotal case reports document acute massive upper and lower gastrointestinal bleeding. ${ }^{21}$ Repeated gastrointestinal bleeding during training and competition may contribute to iron deficiency and anaemia. ${ }^{22}$ Also, endotoxaemia, malabsorption, gastrointestinal tract inflammation, and hypersensitivity reactions have been postulated to occur. ${ }^{13} 142123$ All of these effects, however, can mostly be prevented by appropriate dietary (for example, sufficient fluid intake) and other precautions (for example, avoidance of large amounts of aspirin and non-steroidal anti-inflammatory drugs). ${ }^{12} 22$

In addition to gastrointestinal symptoms, unfavourable effects of exercise on liver function ${ }^{11}$ and peptic ulcer disease $^{24}$ have been reported. However, in well trained endurance-type sportsmen the increase in liver size can be interpreted as a physiological adaptation to increased energy expenditure rather than an expression of liver damage. ${ }^{25}$ Abnormal high serum levels of bilirubin, aspartate aminotransferase, and alkaline phosphatase are seldom observed in blood, underlining the fact that prolonged strenuous exercise does not lead to serious hepatic damage.

Abbreviations used in this paper: RR, relative risk; CD, Crohn's disease; UC, ulcerative colitis; $\dot{\mathrm{VO}}_{2 \max }$, maximal oxygen consumption. 
Only under extreme exercise conditions, such as heat shock, may hepatic damage occur. ${ }^{25}$ In the elderly, long term physical activity even may improve liver function. ${ }^{26}$

Epidemiological studies ${ }^{24} 27$ show that physically demanding occupations may enhance the risk of peptic ulcer disease, independent of several other risk factors such as social class. ${ }^{27}$ However, these studies were hampered by some limitations and pitfalls which question their conclusions. Recently, ${ }^{28}$ no relationship between a history of peptic ulcer and leisure time physical activity was found. Physical activity may even contribute to healing of gastric and duodenal ulcers by normalisation of the microcirculation at the ulcer regions. ${ }^{29}$

\section{Benefits}

The potential benefits of physical activity concern mainly effects on cancer risk, cholelithiasis, gastrointestinal haemorrhage, inflammatory bowel disease, diverticular disease, and constipation.

\section{GASTROINTESTINAL CANCERS}

To date, the risk of oesophageal, bile duct, or gall bladder cancers have not been examined in relation to physical activity. Concerning stomach cancer, the data are controversial: one study reported a reduced risk while two did not. ${ }^{30}$ No relationship between physical activity and risk of pancreatic cancers has been found, ${ }^{30}{ }^{31}$ whereas rectal cancer risk was unrelated to physical activity in the majority of studies. $^{41}$

In contrast, there is overwhelming evidence that physical activity reduces the risk of colon cancer (for example, see World Cancer Research Fund/American Institute for Cancer Research, ${ }^{4}$ Oliveria and Christos, ${ }^{5}$ and Colditz and colleagues $\left.{ }^{6}\right)$. Despite different methods of assessing the amount and different types of physical activity (at work or during leisure time), there is consistent evidence that physically active men and women are at a reduced risk of colon cancer (up to $50 \%$ reduction in incidence). ${ }^{6}$ This effect is independent of other risk factors such as diet and body weight characteristics. The types of activity that may be of benefit in preventing colon cancer are largely unknown. Studies analysing dose-response relationships suggest that more intense activity may confer greater protection against the risk of colon cancer than less intense activity. $^{56}$

The primary postulated mechanism is that physical activity reduces intestinal transit time which would limit the time of contact between the colon mucosa and cancer promoting contents. Enhancing intestinal transit time may indirectly affect the risk of colon cancer by lowering secondary bile acid concentration or by increasing faecal short chain fatty acids. ${ }^{32}{ }^{33}$ Other mechanisms related to colon cancer risk, such as impaired immune function, specific dietary intake (for example, large amounts of alcohol or fat, low amounts of dietary fibre), or an increase in body mass index, insulin resistance, prostaglandin and triglycerides levels, body iron stores, and/or free radical scavenging enzyme activity, can be altered favourably by physical activity. ${ }^{32}$

\section{CHOLELITHIASIS}

Several studies have been published on the relationship between physical activity and cholelithiasis. While early studies yielded controversial results, most of the later studies suggest a protective effect of physical activity. ${ }^{89}{ }^{34}$ Many of the earlier studies in particular are hampered by several methodological drawbacks: no control for potential risk factors other than age (for example, body weight or diet), small sample sizes, limited methods for physical activity assessment, and low variability in physical activity among subjects. Furthermore, it cannot be ruled out that in some studies the level of physical activity was already reduced as a result of the onset of disease.

Two recent large prospective studies ${ }^{89}$ showed a relative risk (RR) of cholelithiasis of 0.63 in men and 0.69 in women when comparing the most active with the most inactive subjects, whereas sedentary lifestyle (watching television or sitting) resulted in an increased risk ( $R R$ 1.11-3.32). A clear dose-response relationship was observed, independent of several potential risk factors, strongly suggesting that (symptomatic) cholelithiasis can be prevented by physical activity, even beyond its benefit for control of body weight or diet.

The mechanisms by which physical activity may influence the pathogenesis of gall stones are poorly understood but decreased biliary cholesterol secretion, and enhanced gall bladder and colonic motility, all known to be important for gall stone formation at rest, ${ }^{33}$ are postulated. Moreover, many factors which are related to an increased risk of cholesterol gall stone disease, such as glucose tolerance, high serum levels of insulin, triglycerides, and various gall bladder regulatory hormones such as cholecystokinin, and low serum levels of high density lipoprotein cholesterol, are favourably altered by physical activity. ${ }^{33} 356$

\section{GASTROINTESTINAL HAEMORRHAGE}

The only study which examined gastrointestinal haemorrhage and physical activity was a prospective cohort study with three years of follow up in 8205 elderly subjects. ${ }^{37}$ Only severe gastrointestinal haemorrhage was investigated. Physical activity was measured by self reported frequency of walking, gardening, or vigorous physical activity (resulting in sweating) three years before the study baseline. A summary variable for the three activities was also calculated. For those participants doing the activity at least three times per week, RR was significantly lower for walking (0.6) and for the summary variable (0.7) in comparison with sedentary subjects, independent of several other risk factors such as age, sex, mobility, body mass index, or health status. The RR for gardening (0.8) and vigorous physical activity (0.7) was not significantly lower. The authors hypothesised that a relatively increased gastrointestinal blood flow in physically active subjects reduced the risk of gastrointestinal haemorrhage. ${ }^{37}$ One should keep in mind that the findings of this study were restricted to severe haemorrhage in elderly subjects and that no data are available for less severe forms of haemorrhage or for younger subjects.

INFLAMMATORY BOWEL DISEASE

A limited number of studies have investigated the preventive effect of physical activity on Crohn's disease (CD) or ulcerative colitis (UC). Sonnenberg ${ }^{38}$ was the first to show a beneficial effect of physical activity by comparing incidence rates of inflammatory bowel disease among several occupations in 12014 individuals. Sedentary and physically less demanding occupations were associated with a higher risk of inflammatory bowel disease than physically demanding occupations. As in other occupational cross sectional studies, this study may have been hampered by limitations, for instance, less demanding occupations may have attracted the chronically ill and previous occupations were not recorded. Persson and colleagues $^{39}$ confirmed this association in a case control study in patients with CD, but not with UC. They found RR values of 0.6 and 0.5 for weekly and daily exercise, respectively. Since then, inconsistent results have been obtained: one case control study ${ }^{40}$ showed a reduced risk, both for physically active CD and UC patients, while another study in CD patients did not. ${ }^{41}$ Postulated under- 
lying mechanisms were the stress reducing effects of physical activity as well as changes in local neuro-immunoendocrine effects. ${ }^{42}$

While the preventive effect of physical activity remains inconclusive, it has become clear that physical activity is not harmful for patients with inflammatory bowel disease, ${ }^{42}$ despite acute exercise related responses, such as increased serum malondialdehyde levels and activated neutrophils. ${ }^{43}$

Nevertheless, physical activity should be promoted as these patients have muscle weakness and are at risk of osteoporosis. ${ }^{44}$ This risk is especially high with glucocorticoid medication, which causes muscle atrophy and weakness, osteoporosis, and osteopenia. ${ }^{42}$ In addition, physical activity may reduce disease activity and improve physical health, general well being, perceived stress, and quality of life. ${ }^{42}$

\section{DIVERTICULAR DISEASE}

A possible role of physical activity in reducing the risk of diverticular disease was suggested by observations of Manousos and colleagues ${ }^{45}$ who found that diverticular disease was more prevalent among subjects with sedentary occupations than in more active occupations. Recently, Aldoori and colleagues ${ }^{7}$ also observed an inverse relationship with physical activity in a prospective cohort of 47678 American men during four years of follow up. After adjustment for several factors such as dietary fibre intake, overall physical activity reduced the risk of symptomatic diverticular disease (for highest versus lowest extremes, $R R=0.63$ ). Risk reduction was stronger for vigorous activity $(R R=0.60)$ than for non-vigorous activity $(R R=0.93, N S)$. Several specific activities reduced the risk of diverticular disease but only for the combination of jogging and running was the inverse relationship statistically significant. An increase in colonic motor activity via hormonal, vascular, and mechanical aspects, leading to a reduction in colonic transit time, was postulated as an underlying mechanism. ${ }^{7}$

\section{CONSTIPATION}

Several cross sectional studies have shown an inverse independent relationship between constipation and physical activity (for example, see Everhart and colleagues, ${ }^{10}$ Donald and colleagues, ${ }^{46}$ and Kinnunen ${ }^{47}$ ). Also, two case control studies ${ }^{48} 49$ showed that the defecation pattern of runners was "better" (less firm stools, higher defecation frequency, higher stool weight) than in inactive controls, a finding which could not be confirmed by the study of Klauser and colleagues. ${ }^{50}$

Inconsistent results were observed in patients who participated in a physical activity programme. Meshkinpour and colleagues ${ }^{48}$ studied eight constipated patients during four weeks of light physical activity (walking five days a week for one hour a day). The distance walked each day progressively increased from 2.9 to $5.2 \mathrm{~km}$, but constipation did not change. Both Karam and Nies ${ }^{51}$ and Resende and colleagues ${ }^{52}$ showed an improvement in defecation pattern and reduced laxative use after a combined physical activity/nutrition programme in elderly patients but the effect of physical activity alone was not studied. In all of these studies physical activity was of relatively low intensity.

Thus the effect of physical activity on constipation seems likely, but has not been proved. Underlying mechanisms are unclear but a favourable effect on colonic motility, decreased blood flow to the gut, biomechanical bouncing of the gut during running, compression of the colon by abdominal musculature, and increased fibre intake as a result of increased energy expenditure have all been reported..$^{53}$

\section{Biological mechanisms}

As mentioned above, the mechanisms by which exercise and physical activity influence the gastrointestinal tract are poorly understood although decreased gastrointestinal blood flow, increase in gastrointestinal motility, increased mechanical bouncing, and neuro-immuno-endocrine alterations are postulated. ${ }^{11-13}$ However, most of the described mechanisms have only been investigated after acute bouts of exercise. Whether or not these mechanisms are predictive of the long term effects of physical activity remains to be elucidated.

\section{GASTROINTESTINAL BLOOD FLOW}

During exercise, blood will primarily be shunted to the skin and exercising muscles at the expense of the gastrointestinal tract. Rowell and colleagues ${ }^{17}$ found a $60-70 \%$ decrease in splanchnic blood flow in humans exercising at $70 \%$ of maximal oxygen consumption $\left(\dot{\mathrm{VO}}_{2 \max }\right)$. At maximal exercise intensity, splanchnic blood flow may be reduced by about $80 \% .^{55}$

Ischaemic damage has been proposed as a causal mechanism of gastrointestinal bleeding during and after exercise. Although gastrointestinal blood loss is transient, increased faecal $\alpha_{1}$ antitrypsin and lysozyme concentrations after exercise have been found, indicative of local mucosal damage with an inflammatory response. ${ }^{21}$ Transient post-exercise lesions from the stomach to the colon have been observed in athletes and the histological picture indicates ischaemic damage. ${ }^{314}$ Critical ischaemic levels may be reached under extreme exercise conditions when hyperthermia, hypohydration, hypoglycaemia, hypoxia, or a combination of these factors are present. ${ }^{13}$ In addition, changes in blood viscosity, erythrocyte deformability, and aggregability during exercise ${ }^{56}$ may further compromise local blood flow. Epithelial cells become deprived of metabolic substrates leading to necrosis and mucosal bleeding.

In theory, critical ischaemic levels and accumulation of metabolic waste products may induce malabsorption, hypersecretion, and increased gastrointestinal permeability with endotoxaemia. Findings for the effect of exercise on absorption and acid secretion are inconsistent: both a decrease or no change in absorption ${ }^{57}$ and acid secretion ${ }^{58} 59$ have been reported. Exercise induced increases in intestinal permeability and mild leakage of endotoxins into the portal circulation have only been found at higher intensities. ${ }^{6061}$

GASTROINTESTINAL MOTILITY AND BILE SALT METABOLISM Data on the effect of exercise and physical activity on gastrointestinal motility are scarce and mostly indirectly obtained and limited to acute exercise. ${ }^{2062} 63$ The direct effect of exercise on gastrointestinal motility has been hypothesised to explain gastrointestinal symptoms such as heartburn (gastro-oesophageal reflux), vomiting, gastrointestinal cramps, urge to defecate, and diarrhoea. ${ }^{13}$

Gastric emptying of liquids and solids appears to be unchanged or slightly accelerated at lower exercise intensities. Above $70 \% \dot{\mathrm{VO}}_{2 \max }$, gastric emptying is certainly delayed. ${ }^{18} 5964$ The long term effects of gastric emptying are inconsistent: Carrio and colleagues ${ }^{65}$ found that basal gastric emptying was faster in runners than in controls while Rehrer et al found no difference. ${ }^{64}$

Up to now two studies have focused on the effects of exercise and physical activity on gall bladder motility. An acute bout of aerobic exercise increased gall bladder ejection fraction slightly but insignificantly in a healthy non-obese female population. ${ }^{66}$ Also, the effect of physical activity was insignificant. In 27 obese female subjects who exercised ( 45 minute brisk walking five times per week) for 12 weeks, postprandial gall bladder ejection fraction 
increased significantly, but this increase, although larger, did not differ from that in the control group. ${ }^{67}$

Orocaecal transit time was reported to be unchanged, delayed, or accelerated ${ }^{20} 5368$ after exercise. Harris and colleagues ${ }^{69}$ found that a high energy intake, which is probably correlated with intensive physical activity, was significantly correlated with fast orocaecal transit.

Studies of the effect of physical activity on colonic transit time have mostly been conducted in healthy subjects and their results are conflicting, probably due to methodological problems: four studies found accelerated transit time after physical activity ${ }^{53} 7071$ while three observed no overall effect. $^{72-74}$ Resende and colleagues ${ }^{52}$ observed no significant changes in gastrointestinal transit time in 12 immobile long stay patients after 12 weeks of individual sessions of massage and physical activity (five days a week, once a day).

Research on the effect of exercise and physical activity on bile salt metabolism is mainly limited to animal studies. These studies showed that physical activity of moderate intensity increased bile acid excretion. ${ }^{75-77}$ Part of these changes were due to hyperphagia. ${ }^{75}$ Research on the effect of physical activity and exercise on secondary bile acid formation and absorption in humans is scarce. In obese patients, a decrease in the secondary/primary bile acid ratio has been observed after treatment with a subcaloric diet and graded physical activity. ${ }^{78}$ Sutherland and colleagues ${ }^{79}$ found that total faecal bile acid concentration was significantly lower in male distance runners than in sedentary men, while bile acid secretion was not different between groups. The lower faecal bile acid concentrations were mainly due to the higher fibre intake and subsequently higher stool weight in runners.

MECHANICAL BOUNCING

The frequency of most gastrointestinal symptoms is almost twice as high during running than during other endurance sports such as cycling or swimming, where up and down movements are more limited. ${ }^{12}$ The mechanical vibration of the body is more than doubled in running compared with cycling. ${ }^{80}$ The way in which this bouncing of the gut affects gastrointestinal function is still unknown. ${ }^{12} 53$

\section{NEURO-IMMUNO-ENDOCRINE ALTERATIONS}

Many hormones associated with gastrointestinal function at rest (secretion, absorption, and motility) alter during exercise, in terms of plasma concentrations: cholecystokinin, vasoactive intestinal peptide, secretin, pancreatic polypeptide, somatostatin, peptide histidine isoleucine, peptide YY, gastrin, glucagon, motilin, catecholamines, endorphins, and prostaglandins. ${ }^{138182}$ However, direct proof that these hormones alter gut function during exercise or induce exercise related gastrointestinal symptoms is lacking.

With respect to immune function, the current opinion is that physical activity of moderate intensity may protect against infections by inducing changes in the activity of macrophages, natural killer cells, lymphokine activated killers cells, neutrophils, and regulating cytokines. ${ }^{32}$ Severe exercise, however, can result in a transient reduction in natural killer cells and production of free radicals, which temporarily increases the risk of infection. ${ }^{32}{ }^{83}$ Knowledge of optimal intensity and duration of physical activity for an optimal immunomodulating effect in athletes and in patients with gastrointestinal related diseases is lacking.

\section{Conclusions}

Strenuous exercise may induce gastrointestinal symptoms such as heartburn or diarrhoea, which may deter people from participating in physical activity. Although many

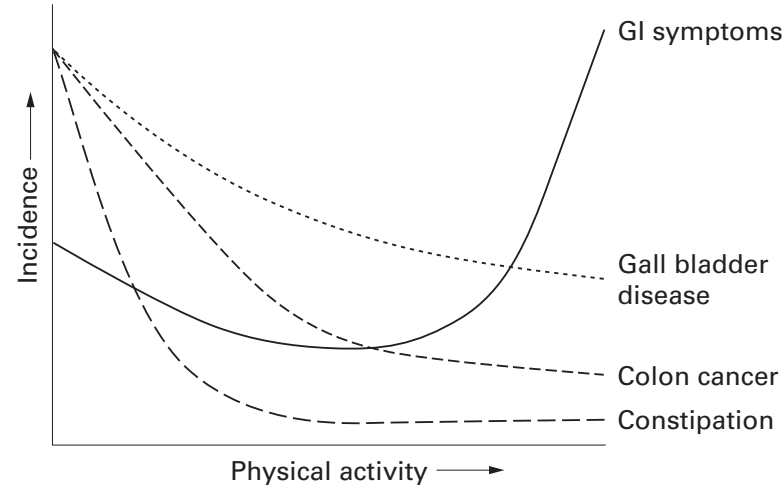

Figure 1 Putative relationship between the incidence of some gastrointestinal diseases/symptoms and amount of physical activity, ranging from bed rest to marathon running or triathlon. The relationship of other gastrointestinal diseases (that is, diverticulosis, gastrointestinal haemorrhage, inflammatory bowel disease, peptic ulcer disease) is not depicted due to limited evidence.

symptoms are acute and transient and do not hamper the athlete's health, repeated gastrointestinal bleeding during training and competition may occasionally lead to iron deficiency and anaemia. However, these and other symptoms can often be prevented with appropriate precautions.

Physical activity, mostly performed at a relatively low intensity, may also have protective effects on the gastrointestinal tract. There is strong evidence that physical activity reduces the risk of colon cancer. Less convincing evidence is found for cholelithiasis and constipation. The putative relationship between the incidence of these gastrointestinal diseases/symptoms and amount of physical activity is shown in fig 1 . Physical activity may reduce the risk of diverticulosis, gastrointestinal haemorrhage, and inflammatory bowel disease, although up to now there has been little research to substantiate this. Physical activity does not interfere with the healing process in inflammatory bowel disease and will probably not reduce the risk of rectal and gastric cancer.

Future research on exercise associated digestive processes in health or disease should explore the mechanisms involved in the potential benefits and hazards of physical activity and exercise on the gastrointestinal tract.

H P F PETERS W R DE VRIES

Department of Medical Physiology and Sports Medicine,

University Medical Centre Utrecht, Utrecht, The Netherlands

\section{G P VANBERGE-HENEGOUWEN} L M A AKKERMANS

Gastrointestinal Research Unit,

Departments of Surgery and Gastroenterology,

University Medical Centre Utrecht, Utrecht, The Netherlands

Correspondence to: Dr H P F Peters, Department of Medical Physiology and Sports Medicine, University Medical Centre Utrecht, PO Box 85060, 3508

AB Utrecht, The Netherlands.

h.p.f.peters@med.uu.nl

Sullivan SN. Exercise-associated symptoms in triathletes. Phys Sports Med 1987;15:105-8.

2 Rehrer NJ, Janssen GM, Brouns F, et al. Fluid intake and gastrointestinal problems in runners competing in a $25-\mathrm{km}$ race and a marathon. Int $\mathcal{F}$ Sports Med 1989;10(suppl 1):S22-5.

3 Moses FM, Brewer TG, Peura DA. Running-associated proximal hemorrhagic colitis. Ann Intern Med 1988;108:385-6.

4 Food, nutrition and the prevention of cancer: a global perspective. World Cancer Research Fund/American Institute for Cancer Research, 1997;216-24.

5 Oliveria SA, Christos PJ. The epidemiology of physical activity and cancer. Ann N Y Acad Sci 1997;833:79-90.

6 Colditz GA, Cannuscio CC, Frazier AL. Physical activity and reduced risk of colon cancer: implications for prevention. Cancer Causes Control 1997;8: 649-67.

7 Aldoori WH, Giovannucci EL, Rimm EB, et al. Prospective study of physical activity and the risk of symptomatic diverticular disease in men. Gut 1995;36:276-82. 
8 Leitzmann MF, Rimm EB, Willett WC, et al. Recreational physical activity and the risk of cholecystectomy in women. N Engl F Med 1999;341:777-84 Leitzmann MF, Giovannucci EL, Rimm EB, et al. The relation of physical activity to risk for symptomatic gallstone disease in men. Ann Intern Med 1998;128:417-25.

10 Everhart JE, Go VL, Johannes RS, et al. A longitudinal survey of self-reported bowel habits in the United States. Dig Dis Sci 1989;34:115362.

11 Moses FM. The effect of exercise on the gastrointestinal tract. Sports Med 1990;9:159-72.

12 Peters HP, Akkermans LM, Bol E, et al. Gastrointestinal symptoms during exercise. The effect of fluid supplementation. Sports Med 1995;20:65-76.

13 Brouns F, Beckers E. Is the gut an athletic organ? Digestion, absorption and exercise. Sports Med 1993;15:242-57.

14 Øktedalen O, Lunde OC, Opstad PK, et al. Changes in the gastrointestinal mucosa after long-distance running. Scand f Gastroenterol 1992;27:270-4.

15 Peters HP, Bos M, Seebregts L, et al. Gastrointestinal symptoms in long-distance runners, cyclists, and triathletes: prevalence, medication, and etiology. Am f Gastroenterol 1999:94:1570-81.

16 Peters HP, Zweers M, Backx FJ, et al. Gastrointestinal symptoms during long-distance walking. Med Sci Sports Exerc 1999;31:767-73.

17 Rowell LB, Blackmon JR, Bruce RA. Indocyanine green clearance and estimated hepatic blood flow during mild to maximal exercise in upright man. f Clin Invest 1964;43:1677-90

18 Neufer PD, Young AJ, Sawka MN. Gastric emptying during walking and running: effects of varied exercise intensity. Eur $\mathcal{F}$ Appl Physiol 1989;58:4405.

19 Soffer EE, Wilson J, Duethman G, et al. Effect of graded exercise on esophageal motility and gastroesophageal reflux in nontrained subjects. Dig Dis Sci 1994;39:193-8.

20 Soffer EE, Summers RW, Gisolfi C. Effect of exercise on intestinal motility and transit in trained athletes. Am F Physiol 1991;260:G698-702.

21 Peters HP, Wiersma WC, Akkermans LM, et al. Gastrointestinal mucosal integrity after prolonged exercise with fluid supplementation. Med Sci Sports Exerc 2000;32:134-42.

22 Nielsen P, Nachtigall D. Iron supplementation in athletes. Current recommendations. Sports Med 1998;26:207-16.

23 Bosenberg AT, Brock-Utne JG, Gaffin SL, et al. Strenuous exercise causes systemic endotoxemia. $\mathcal{F}$ Appl Physiol 1988;65:106-8.

24 Sonnenberg A. Factors which influence the incidence and course of peptic ulcer. Scand $\mathcal{f}$ Gastroenterol Suppl 1988;155:119-40.

25 Ritland S, Foss NE, Gjone E. Physical activity in liver disease and liver function in sportsmen. Scand F Soc Med Suppl 1982;29:221-6.

26 Mauriz JL, Tabernero B, Garcia-Lopez J, et al. Physical exercise and improvement of liver oxidative metabolism in the elderly. Eur $\mathcal{F}$ Appl Physiol 2000;81:62-6.

27 Katschinski BD, Logan RF, Edmond M, et al. Physical activity at work and duodenal ulcer risk. Gut 1991;32:983-6.

28 Suadicani P, Hein HO, Gyntelberg F. Genetic and life-style determinants of peptic ulcer. A study of 3387 men aged 54 to 74 years: The Copenhagen Male Study. Scand $\mathcal{F}$ Gastroenterol 1999;34:12-17.

29 Efremushkin GG, Titova ZA, Molchanov AV, et al. The effect of combined treatment using bicycle exercise with a free choice of the load parameters on the hemodynamics in peptic ulcer patients (in Russian). Ter Arkh 1998; 70:13-16.

30 Lee IM. Physical activity, fitness, and cancer. In: Bouchard C, Shephard RJ, Stephens T, eds. Physical activity, fitness, and health. International proceedings and consensus statement. Champaign, IL: Human Kinetics, 1994:814-31.

31 Lee IM, Paffenbarger RSJ. Physical activity and its relation to cancer risk: a prospective study of college alumni. Med Sci Sports Exerc 1994;26:831-7.

32 Shephard RJ, Shek PN. Associations between physical activity and susceptibility to cancer: possible mechanisms. Sports Med 1998;26:293-315.

33 Erpecum Van K, Van Berge-Henegouwen GP. Gallstones: an intestinal disease? Gut 1999;44:435-8.

34 Rissanen A, Fogelholm M. Physical activity in the prevention and treatment of other morbid conditions and impairments associated with obesity:
current evidence and research issues. Med Sci Sports Exerc 1999;31:S63545 .

35 Anonymous. The epidemiology of gallstone disease in Rome, Italy. Part II. Factors associated with the disease. The Rome Group for Epidemiology and Prevention of Cholelithiasis (GREPCO). Hepatology 1988;8:907-13.

36 Bennion LJ, Grundy SM. Risk factors for the development of cholelithiasis in man. N Engl f Med 1978;299:1161-7.

37 Pahor M, Guralnik JM, Salive ME, et al. Physical activity and risk of severe gastrointestinal hemorrhage in older persons. $7 A M A$ 1994;272:595-9.

38 Sonnenberg A. Occupational distribution of inflammatory bowel disease among German employees. Gut 1990;31:1037-40.

39 Persson PG, Leijonmarck CE, Bernell O, et al. Risk indicators for inflammatory bowel disease. Int $\mathcal{F}$ Epidemiol 1993;22:268-72.

40 Klein I, Reif S, Farbstein H, et al. Preillness non dietary factors and habits in inflammatory bowel disease. Ital f Gastroenterol Hepatol 1998;30:247-51.

41 Sørensen VZ, Olsen BG, Binder V. Life prospects and quality of life in patients with Crohn's disease. Gut 1987;28:382-5.

42 Loudon CP, Corroll V, Butcher J, et al. The effects of physical exercise on patients with Crohn's disease. Am f Gastroenterol 1999;94:697-703.

43 D'Inca R, Varnier M, Mestriner C, et al. Effect of moderate exercise on Crohn's disease patients in remission. Ital f Gastroenterol Hepatol 1999;31: 205-10.

44 Robinson RJ, Krzywicki T, Almond L, et al. Effect of a low-impact exercise program on bone mineral density in Crohn's disease: a randomized controlled trial. Gastroenterology 1998;115:36-41

45 Manousos ON, Vrachliotis G, Papaevangelou G, et al. Relation of diverticulosis of the colon to environmental factors in Greece. Am F Dig Dis 1973; 18:174-6.
46 Donald IP, Smith RG, Cruikshank JG, et al. A study of constipation in the elderly living at home. Gerontology 1985;31:112-18.

47 Kinnunen O. Study of constipation in a geriatric hospital, day hospital, old people's home and at home. Aging 1991;3:161-70.

48 Meshkinpour H, Selod S, Movahedi H, et al. Effects of regular exercise in management of chronic idiopathic constipation. Dig Dis Sci 1998;43:237983

49 Sullivan SN, Wong C, Heidenheim P. Does running cause gastrointestinal symptoms? A survey of 93 randomly selected runners compared with controls. N Z Med F 1994;107:328-31.

50 Klauser AG, Peyerl C, Schindlbeck NE, et al. Nutrition and physical activity in chronic constipation. Eur f Gastroenterol Hepatol 1992;4:227-33.

51 Karam SE, Nies DM. Student/staff collaboration: a pilot bowel management program. I Gerontol Nurs 1994;20:32-40.

52 Resende TL, Brocklehurst JC, O’Neill PA. A pilot study on the effect of exercise and abdominal massage on bowel habit in continuing care patients. Clin Rehabil 1993; 7:204-9.

53 Koffler KH, Menkes A, Redmond RA, et al. Strength training accelerates gastrointestinal transit in middle-aged and older men. Med Sci Sports Exerc 1992;24:415-19.

54 Oettlé GJ. Effect of moderate exercise on bowel habit. Gut 1991;32:941-4.

55 Clausen JP. Effect of physical training on cardiovascular adjustments to exercise in man. Physiol Rev 1977;57:779-815.

56 Vandewalle $\mathrm{H}$, Lacombe C, Lelievre JC, et al. Blood viscosity after a 1-h submaximal exercise with and without drinking. Int $\mathcal{f}$ Sports Med
1988;9:104-7.

57 Schedl HP, Maughan RJ, Gisolfi CV. Intestinal absorption during rest and exercise: implications for formulating an oral rehydration solution (ORS). Proceedings of a roundtable discussion. April 21-22, 1993. Med Sci Sports Exerc 1994;26:267-80.

58 Markiewicz K, Lukin M. Maximal gastric secretion during physical exertion and restitution in patients with chronic duodenal ulcer (in Polish). Pol Arch Med Wewn 1988;79:13-19.

59 Feldman M, Nixon JV. Effect of exercise on postprandial gastric secretion and emptying in humans. $\mathcal{F}$ Appl Physiol 1982;53:851-4.

60 Pals KL, Chang R-T, Ryan AJ, et al. Effect of running intensity on intestinal permeability. F Appl Physiol 1997;82:571-6.

61 Jeukendrup AE, Vet-Joop K, Sturk A, et al. Relationship between gastro-intestinal complaints and endotoxaemia, cytokine release and the acute-phase reaction during and after a long-distance triathlon in highly trained men. Clin Sci 2000;98:47-55.

62 Rao SS, Beaty J, Chamberlain M, et al. Effects of acute graded exercise on human colonic motility. Am f Physiol 1999;276:G1221-6.

63 Cheskin LJ, Crowell MD, Kamal N, et al. The effects of acute exercise on colonic motility. $\mathcal{F}$ Gastrointest Motil 1992;4:173-7.

64 Rehrer NJ, Beckers E, Brouns F, et al. Exercise and training effects on gastric emptying of carbohydrate beverages. Med Sci Sports Exerc 1989;21: $540-9$.

65 Carrio I, Estorch M, Serra-Grima R, et al. Gastric emptying in marathon runners. Gut 1989;30:152-5.

66 Utter AC, Goss FL, Whitcomb DC, et al. The effects of acute exercise on gallbladder function in an adult female population. Med Sci Sports Exerc 1996;28:280-4.

67 Utter AC, Whitcomb DC, Nieman DC, et al. Effects of exercise training on gallbladder function in an obese female population. Med Sci Sports Exerc 2000;32:41-5.

68 Keeling WF, Harris A, Martin BJ. Loperamide abolishes exercise-induced orocecal liquid transit acceleration. Dig Dis Sci 1993;38:1783-7.

69 Harris A, Lindeman AK, Martin BJ. Rapid orocecal transit in chronically active persons with high energy intake. f Appl Physiol 1991;70:1550-3.

70 Cordain L, Latin RW, Behnke JJ. The effects of an aerobic running program on bowel transit time. $\mathcal{F}$ Sports Med Phys Fitness 1986;26:101-4.

71 Liu F, Kondo T, Toda Y. Brief physical inactivity prolongs colonic transit time in elderly active men. Int $\mathcal{F}$ Sports Med 1993;14:465-7.

72 Bingham SA, Cummings JH. Effect of exercise and physical fitness on large intestinal function. Gastroenterology 1989;97:1389-99.

73 Coenen C, Wegener M, Wedmann B, et al. Does physical exercise influence bowel transit time in healthy young men? Am f Gastroenterol 1992;87:292-

74 Robertson G, Meshkinpour H, Vandenberg K, et al. Effects of exercise on total and segmental colon transit. F Clin Gastroenterol 1993;16:300-3.

75 Yiamouyiannis CA, Martin BJ, Watkins JB3. Chronic physical activity alters hepatobiliary excretory function in rats. F Pharmacol Exp Ther 1993;265: 321-7.

76 Watkins JB, Crawford ST, Sanders RA. Chronic voluntary exercise may alter hepatobiliary clearance of endogenous and exogenous chemical in rats. 994;22:537-43.

77 Bouchard G, Carrillo MC, Tuchweber B, et al. Moderate long-term physical activity improves the age-related decline in bile formation and bile salt secretion in rats. Proc Soc Exp Biol Med 1994;206:409-15.

78 Kadyrova RK, Shakieva RA. Dynamics of changes in the lipid composition of bile in patients with alimentary obesity during treatment (in Russian). Ter Arkh 1986;58:79-82

79 Sutherland WHF, Nye ER, Macfarlane DJ, et al. Fecal bile acid concentration in distance runners. Int $\mathcal{F}$ Sports Med 1991;12:533-6.

80 Rehrer NJ, Meijer GA. Biomechanical vibration of the abdominal region during running and bicycling. F Sports Med Phys Fitness 1991;31:231-4.

81 O'Connor AM, Johnston CF, Buchanan KD, et al. Circulating gastrointestinal hormone changes in marathon running. Int 7 Sports Med 1995; 16:283-7.

82 Demers LM, Harrison TS, Halbert DR, et al. Effect of prolonged exercise on plasma prostaglandin levels. Prostaglandins Med 1981;6:413-18.

83 Nieman DC. Immune response to heavy exertion. If Appl Physiol 1997;82:1385-94. 